\title{
Application of PID Neural Network in the pneumatic control system
}

\author{
PAN Yu Xuan ${ }^{1, a}$, DONG Quan Lin ${ }^{1, b}$ \\ ${ }^{1}$ BEIHANG University Beijing 100191, China \\ apanyuxuan3743@163.com, ${ }^{\text {bdongquanlin@buaa.edu.cn }}$
}

Keywords: intelligent valve positioner, pneumatic servo system, PID neural network, regulating valve, nonlinear control

Abstract. In order to solve the problems of time-varying, nonlinear and large inertia in the pneumatic control system of the intelligent valve positioner, the PID neural network control strategy is used to control the valve opening. Based on the analysis of the principle of the neural network controller, the network structure and the formulas of the PID neural network controller are determined to improve the system control accuracy and the dynamic performance. The simulation results show that the PID neural network has better time-varying, less adjustment time and greater accuracy than the traditional PID. The experimental results show that the settling time of the control strategy is less than $4 \mathrm{~s}$ and the accuracy of the strategy is less than $1 \%$.

\section{Introduction}

Because the valve control cylinder system as the control object of the valve positioner has the characteristics of time-varying and nonlinear, it is difficult to obtain an accurate mathematical model in the modeling analysis. Parameters of the PID can't be adjusted in real time, so it is difficult to achieve the excellent effect of the valve opening control by the PID algorithm [1,5]. Neural network does not need the accurate mathematical model. The paper uses the characteristics of positive learning and reverse correction of the neural network to control the object. In order to make the valve opening control have ideal steady-state characteristics and dynamic characteristics, this paper combines the neural network and the PID to realize the real-time adjustment of PID parameters, the optimization of the positioning performance of the intelligent valve and the enhancement of the positioning accuracy.

The paper introduces the composition and working principle of pneumatic servo system, and provides an effective theoretical reference for the research of control strategy. Then, the control strategy of pneumatic control is studied, and the conventional PID control algorithm based on compound neural network is used to improve the precision of valve position closed-loop control. Finally, the experimental platform of the control system is established, and the function modules are analyzed thoroughly.

\section{Valve Opening Control System}

The valve opening control system is a closed loop pneumatic servo system. The system uses compressed air as the final control medium to control the valve core displacement. And the displacement and the speed of the valve core are as the feedback object. The pneumatic servo system which is an embedded system can use the PWM to control the conversion from electrical signals to gas signals. Its course of work is as follow: after the MCU receives the external command, it can judge whether the current valve is consistent with the set value. If the set value and the feedback value is different, the system output a certain duty cycle PWM signal and solenoid valves are driven in a number of cycles of inflatable or deflated by the PWM signal, so that pressures in two chambers of the actuator are different. The actuator piston is moved by the change of the pressure and drives the valve core to reach the command position. The structure of the servo system is shown in Fig. 1. 


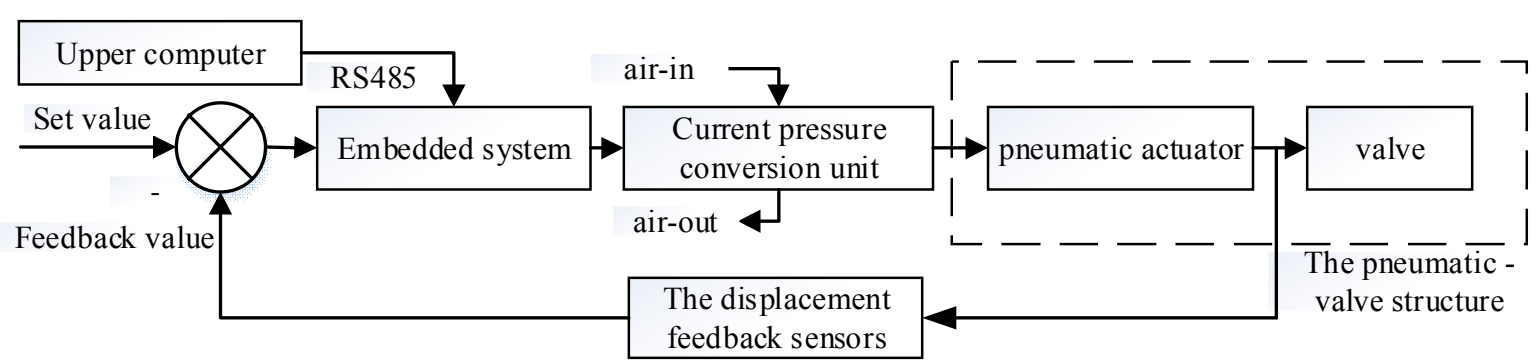

Fig.1 The structure of the servo system

\section{PID neural network control algorithm}

PID neural network algorithm is one of the algorithms to realize the real-time adjustment of PID parameters, which has the characteristics of simple structure, fast speed and good robustness[6,7]. As shown in Fig. 2, the PID neural network can be divided into the input layer, the hidden layer and the output layer. In the single output neural network, the current value and the target value of the object are used as the inputs, and the hidden layer includes three parameters of the PID. As shown in Fig. 2, $X 1$ is the input target value. $X 2$ is the current value. $Y$ is the control rate of the output. $\omega_{i j}$ and $\omega_{j k}$ are the weighted values.

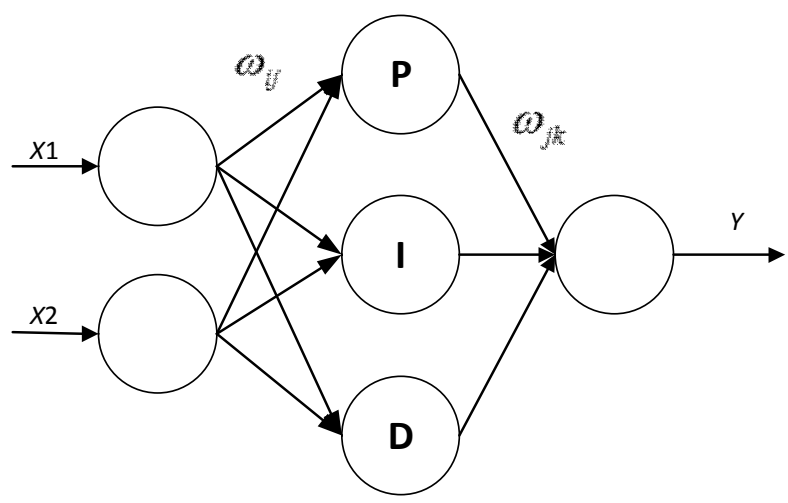

Fig.2 Single output PID neural network structure

\section{PID neural network control rate calculation}

The formulas of the input layer and the hidden layer of the neural network are as follows:

$$
\begin{aligned}
& x_{s i}(k)=X_{s i}(k) . \\
& n e t_{s i}(k)=\sum_{i=1}^{2} \omega_{i j} x_{s i}(k) \quad j=1,2,3 .
\end{aligned}
$$

The proportion of neurons, integral neurons, and differential neurons are calculated as follows:

$$
\begin{aligned}
& u_{s 1}(k)=n e t_{s 1}(k) . \\
& u_{s 2}(k)=n e t_{s 2}(k)+u_{s 2}(k-1) . \\
& u_{s 3}(k)=n e t_{s 3}(k)-n e t_{s 3}(k-1) .
\end{aligned}
$$

where, $s$ is the parallel subnetwork number; $j$ is the number of the hidden layer element in the subnetwork; $x_{s i}(k)$ is the input value of each subnet input layer; $\omega_{i j}$ is the weighted value from the input layer to the hidden layer.

The output layer has n neurons, which is the weighted sum of the output values of all neurons in the hidden layer. The formula is as follow: 


$$
y_{h}(k)=\sum_{s=1}^{n} \sum_{j=1}^{3} \omega_{i j} u_{s j}(k)
$$

where, $h$ is the output neuron number; $u_{s j}(k)$ is the output value of each neuron of the hidden layer; $\omega_{j k}$ is the weighted value from the hidden layer to the output layer.

\section{PID neural network weight correction}

In the process of realizing the control, the PID neural network adjusts the weight with the error of the control quantity according to the gradient correction method so that the control quantity is close to the control target value.

The process of weight correction is as follows:

$$
J=\sum E=\sum_{k=1}^{n}\left[y_{h}(k)-r(k)\right]^{2}
$$

where, $n$ is the number of output nodes; $y_{h}(k)$ is the input string with the layer; $r(k)$ is the control target.

The weight correction formulas from the output layer to the hidden layer and from the input layer to the output layer are discrete as follows:

$$
\begin{aligned}
& \omega_{j k}(k+1)=\omega_{j k}(k)-\eta \frac{\partial J}{\partial \omega_{j k}} . \\
& \omega_{i j}(k+1)=\omega_{i j}(k)-\eta \frac{\partial J}{\partial \omega_{i j}} .
\end{aligned}
$$

where, $\eta$ is the learning rate.

\section{Simulation analysis}

In order to compare the difference between PID and PID neural network, parameters of the PID should be determined. As shown in Fig. 3, a signal constraint module is added to the existing simulink model and connected to the output signal to constrain the output response of the PID. The module can convert the time domain constraints into the optimal constraints. $K_{P}, K_{I}$ and $K_{D}$ are added as optimization parameters to the workspace and set the range of parameters. And the optimization algorithm in the Optimization Toolbox is used to solve.

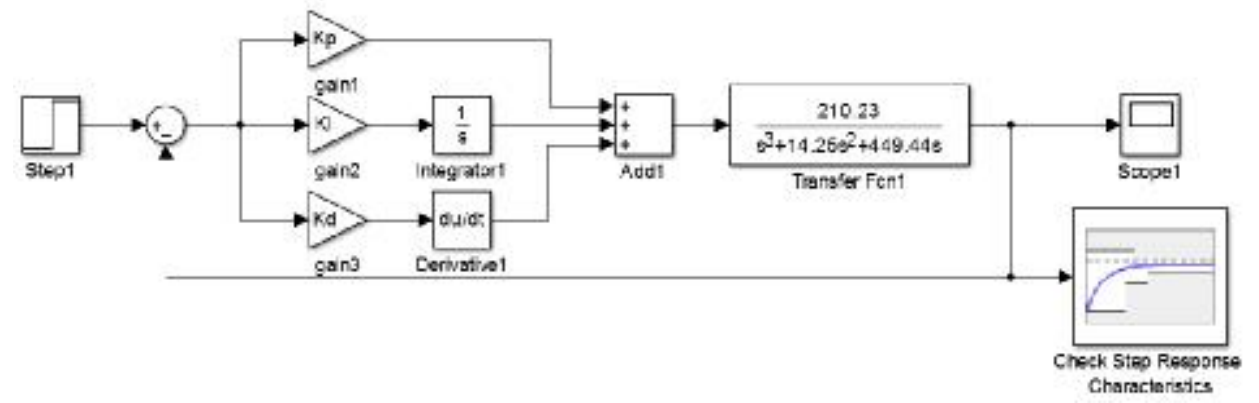

Fig.3 PID parameters optimization Signal Constraint module

Overshoot less than $20 \%$, rise time $1 \mathrm{~s}$, settling time $3 \mathrm{~s}$ and error range $\pm 0.5 \%$ are the parameters of the optimization conditions. The optimized PID parameters are $K_{p 0}=11.3098, K_{i 0}=19.2136$, $K_{d 0}=0.0220$.

\section{Improvement of PID neural network controller}

The conventional gradient learning formula of PID neural network may cause the weight correction to be slow and trap into local optimum. Therefore, the method of increasing momentum is used to 
improve the efficiency of network learning. The formulas for increasing the momentum are as following:

$$
\omega_{i j}(k+1)=\omega_{i j}(k)-\eta \frac{\partial J}{\partial \omega_{i j}}+\eta_{1}\left[\omega_{i j}(k)-w_{i j}(k-1)\right] .
$$

where, $w_{i j}(k)$ is the control error, $\eta_{1}$ is the learning rate.

In order to prevent the differential neuron value is too small and the integral neuron value is too large, the parameter settings in PID algorithm are used to rise the product coefficient of neuron output. The improved Eqs.(4) (5) and (6) are as following:

$$
\begin{aligned}
& u_{s 1}(k)=k_{p} \bullet \operatorname{net}_{s 1}(k) . \\
& u_{s 2}(k)=k_{i} \bullet \operatorname{net}_{s 2}(k)+k_{i} \bullet u_{s 2}(k-1) . \\
& u_{s 3}(k)=k_{d} \bullet \operatorname{net}_{s 3}(k)-k_{d} \bullet n e t_{s 3}(k-1) .
\end{aligned}
$$

\section{Simulation comparison experiment}

The simulation model of effect contrast between PID neural network and conventional PID is built in MATLAB. In simulation, the scale factor and the quantization factor are adjusted for obtaining the best effect. The scale factor and the quantization factor are less than 1.

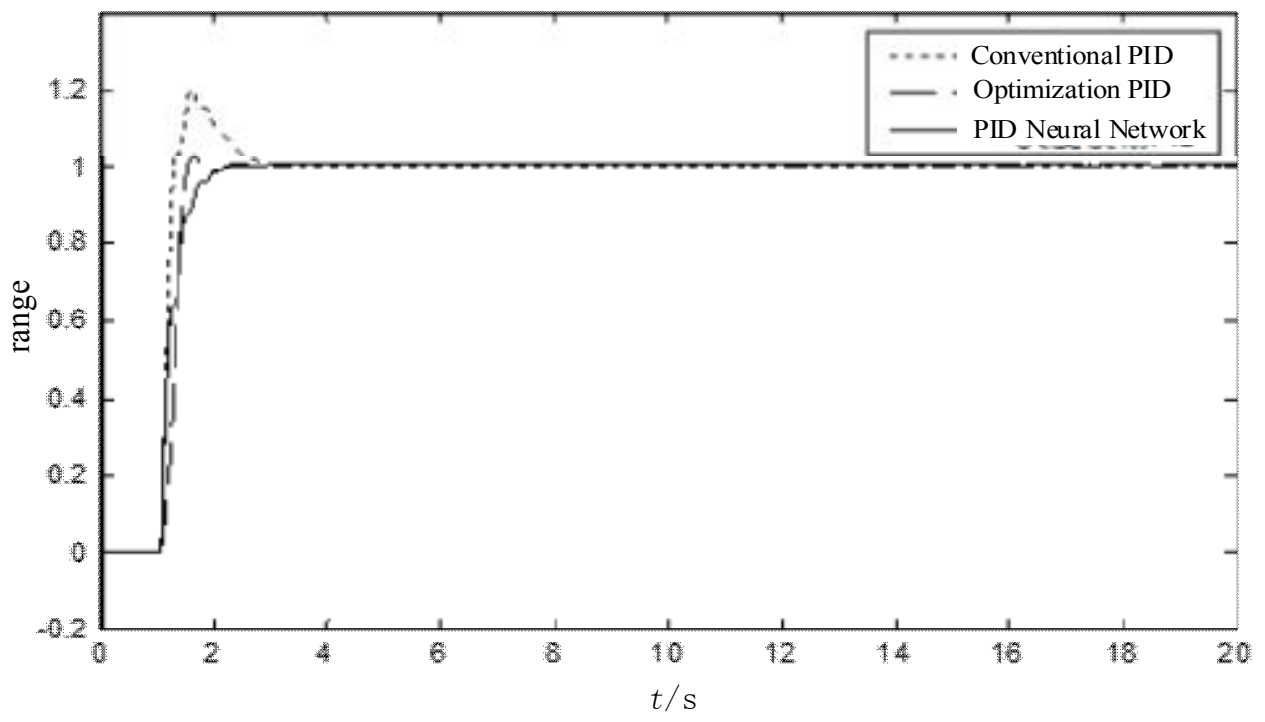

Fig.4 system step response curve

The response curve of the Fig. 4 can be used to compare the performance of the two algorithms as shown in table 1:

Table 1 Performance index of system step response

\begin{tabular}{ccc}
\hline Control algorithm & Overshoot & Settling time(s) \\
\hline PID & $20 \%$ & 3.0 \\
PID neural network & $0.05 \%$ & 2.4 \\
\hline
\end{tabular}

Simulation results show that when the PID is used to control the object with time-varying and non-linear links, the response efficiency is low. The control effect is not ideal. The control law of the control system can be selected by the following characteristics:

1) If there is a large lag in the controlled system and the effect of time on the load is significant, the PID algorithm should be selected;

2) If there are multiple inertia links and hysteresis links in the system and the load is time-varying. PID neural network should be adopted. 


\section{Experimental verification}

\section{Introduction to experimental devices}

The experimental device used in the debugging process is used to test the control performance of the intelligent positioning valve. It is mainly composed of pipeline, water pump, water source, gas source, valves and data acquisition system. The device can achieve the intelligent positioning valve on the control of different valves in different pipelines, different water pressure and pressure.

\section{Experimental results analysis}

The experiment of the intelligent positioning valve is carried out under the condition of fully simulating the field condition. The equipment parameters are shown in Table 2.

Table 2 Parameters of system experiment equipment

\begin{tabular}{ll}
\hline Experimental equipment & Parameter \\
\hline Gas source(MPa) & 0.6 to 1 \\
Pneumatic control valve & Range $15 \mathrm{~mm}$, less than $1 \mathrm{Mpa}$ \\
Signal generator & Provide 4to20mA current \\
Pipe diameter & DN50 \\
Water pressure $(\mathrm{MP} a)$ & 0.8 to 1.7 \\
\hline
\end{tabular}

During the experiment, the gas source and water source is firstly opened. The outputs of air pressure and water pressure are adjusted to $0.6 \mathrm{Mpa}$ and $0.8 \mathrm{MPa}$. Then the signal generator is used to impose different opening control instructions to the system, while the corresponding data are recorded by the acquisition system. In order to explore the distribution range of system control accuracy under different working conditions, five representative points of $0 \%, 25 \%, 50 \%, 75 \%$ and $100 \%$ of the opening are tested in the experiment. The water pressure range is settled to 0.8 to $1.7 \mathrm{MPa}$ at each point of the test. 10 sets of data are tested at each intervals of $0.1 \mathrm{MPa}$. The collected data in the experimental process are shown in Table 3.

Table 3 Test results of valve opening under different water pressure

\begin{tabular}{rc|ccccc}
\hline Set value & & $0 \%$ & $25 \%$ & $50 \%$ & $75 \%$ & $100 \%$ \\
\hline & 0.8 & $0.76 \%$ & $25.67 \%$ & $49.39 \%$ & $74.25 \%$ & $99.09 \%$ \\
& 0.9 & $0.76 \%$ & $25.68 \%$ & $49.40 \%$ & $74.24 \%$ & $99.11 \%$ \\
& 1.0 & $0.77 \%$ & $25.68 \%$ & $49.39 \%$ & $74.25 \%$ & $99.12 \%$ \\
Actual value in & 1.1 & $0.76 \%$ & $25.68 \%$ & $49.38 \%$ & $74.23 \%$ & $99.12 \%$ \\
different water & 1.2 & $0.78 \%$ & $25.68 \%$ & $49.39 \%$ & $74.27 \%$ & $99.10 \%$ \\
pressure(MPa) & 1.3 & $0.80 \%$ & $25.69 \%$ & $49.39 \%$ & $74.23 \%$ & $99.12 \%$ \\
& 1.4 & $0.79 \%$ & $25.69 \%$ & $49.39 \%$ & $74.26 \%$ & $99.12 \%$ \\
& 1.5 & $0.78 \%$ & $25.68 \%$ & $49.39 \%$ & $74.27 \%$ & $99.14 \%$ \\
& 1.6 & $0.79 \%$ & $25.70 \%$ & $49.40 \%$ & $74.26 \%$ & $99.13 \%$ \\
Feedback average & 1.7 & $0.80 \%$ & $25.70 \%$ & $49.40 \%$ & $74.27 \%$ & $99.13 \%$ \\
\hline
\end{tabular}

The test results show that the actual opening of the valve have a certain error under different water pressure, and the error increases with the increasing of water pressure. In order to describe the characteristic of positioning intuitively, the theoretical position value at different opening are calculated. And the actual position value of the valve opening is obtained according to the average value of the measured opening. The static error of the valve under each opening is calculated and the control precision under the corresponding opening is obtained as shown in Table 4. 
Table 4 Comparison of precision test results

\begin{tabular}{cccccc}
\hline Opening & $0 \%$ & $25 \%$ & $50 \%$ & $75 \%$ & $100 \%$ \\
\hline theoretical position $(\mathrm{mm})$ & 0 & 3.75 & 7.5 & 11.25 & 15 \\
Feedback position $(\mathrm{mm})$ & 0.117 & 3.8535 & 7.4085 & 11.1375 & 14.868 \\
Static error(mm) & 0.117 & 0.1035 & 0.0915 & 0.1125 & 0.132 \\
Control precision & $0.78 \%$ & $0.69 \%$ & $0.61 \%$ & $0.75 \%$ & $0.88 \%$ \\
\hline
\end{tabular}

Compared with the data in Table 4, there are some errors in the positioning of the intelligent positioning valve control system, and when the opening is less than $50 \%$ of the case, the actual position of the system is slightly beyond the theoretical position. When the opening is higher than $50 \%$, the actual position is difficult to reach the theoretical position. That is due to the effect of pre-pneumatic operation of the pneumatic system is better than the latter gas supply. But on the whole the system error is in the allowable range (the target error range less than 1\%). And the system is closer to the middle position, the positioning is more accurate. That also confirms that there is an unavoidable external resistance at both ends of the valve structure. Therefore, the intelligent valve control system which is designed in this paper meets the design precision requirements.

The collection of multiple experimental data show that: The system response time is less than $4 \mathrm{~s}$, and the control process is more stable to meet the fast and stable control standards; and the system control accuracy can meet the precision requirement; in the control process, the system does not exist the phenomenon of excessive overshoot.

\section{Conclusions}

The conclusions are as following:

(1) In-depth study of the neural network control principle, a pneumatic system control algorithm based on PID neural network is proposed to improve the system control precision and the dynamic performance. The algorithm has the characteristics of high control precision and good real-time performance.

(2) The PID neural network is optimized and simulated. The simulation results show that the proposed algorithm has the advantages of short response time, small overshoot, good steady-state performance and good robust performance, and its control performance is superior to conventional PID.

(3) The experimental platform of the whole test of the valve opening control system is set up and the experimental data of the whole system are given. The results show that the control strategy of PID neural network can control the valve opening control system effectively rapidly, and the precision is less than $1 \%$.

\section{References}

[1] Luo Y, Che X, He Z, et al. Grey Self Adaptive Fuzzy Sliding Mode Control for Flight Simulator Servo System[J]. Lecture Notes in Computer Science, 2008:265-274.

[2] Weixing F, Zeming F, Liang W. Self-tuning of PID Parameters on Intelligent Valve Positioner[J]. MAHN OOL \& HYDRAL, 2009.

[3] Lei S, Kaiming S, peng Z. An Intelligent Valve Positioner Based on the Hall Encoder[J]. Techniques of Automation and Applications, 2009.

[4] Ranjbari L, Shirdel A, Aslahi Shahri M, et al. Designing Precision Fuzzy Controller for Load Swing of An Overhead Crane [J]. Neural Computing \& Applications, 2015, 26:1-6.

[5] Shang Qunli, Jiang Peng. Research on intelligent electro-pneumatic valve positioner[J]. Chinese Journal of Scientific Instrument, 2007, 28(4): 718. 
[6] Huang X, Yu F. A Simple Method for Fault Detection of Industrial Digitial Positioners[C]. //Intelligent Control and Automation, 2008. WCICA 2008. 7th World Congress on. IEEE, 2008:6863 - 6866

[7] Huaxiang Y W W. Parameter Setting of The Electro-pneumatic Valve Positioner [J]. Electronic Measurement Technology, 2008, 1: 014. 